\title{
Bulk and surface characterization of a dewetting thin film polymer bilayer
}

\author{
H. Ade, D. A. Winesett, and A. P. Smith \\ Department of Physics, North Carolina State University, Raleigh, North Carolina 27695-8202 \\ S. Anders, T. Stammler, and C. Heske \\ Advanced Light Source, Ernest Orlando Lawrence Berkeley National Laboratory, Berkeley, California 94720 \\ D. Slep \\ Advanced Development and Research, Hilord Chemical Corporation, Hauppauge, New York 11788 \\ M. H. Rafailovich and J. Sokolov \\ Department Materials Science and Engineering, SUNY at Stony Brook, Stony Brook, New York 11794 \\ J. Stöhr \\ IBM Research Division, Almaden Research Center, San Jose, California 95120
}

(Received 15 June 1998; accepted for publication 14 October 1998)

\begin{abstract}
We have monitored the progression of the dewetting of a partially brominated polystyrene (PBrS) thin film on top of a polystyrene (PS) thin film with scanning transmission $\mathrm{x}$-ray microscopy (STXM) as well as photoemission electron microscopy (PEEM). We mapped the projected thickness of each constituent polymer species and the total thickness of the film with STXM, while we determined the surface composition with PEEM. Our data show that the PBrS top layer becomes encapsulated during the later stages of dewetting and that atomic force microscopy topographs cannot be utilized to determine the contact angle between PBrS and PS. (C) 1998 American Institute of Physics. [S0003-6951(98)02351-1]
\end{abstract}

Thin films of polymers have numerous technological applications ranging from multicolor photographic printing to paints, adhesives, index-matched optical coatings, photoresists, and low dielectric electronic packaging. In comparison to bulk properties, much less is known about the properties of polymers when they are processed into thin films. Typically, the polymer-substrate and polymer-air interfacial energies or kinetic barriers play important roles in determining the morphology and dynamics in thin films. A detailed understanding of thin film properties, such as composition and morphology, and possible deviations from bulk properties is highly desirable and numerous studies on blends ${ }^{1-7}$ and bilayers $^{8,9}$ have recently been undertaken. Most of these studies rely on atomic force microscopy (AFM) or other methods that cannot always provide the sought after answers. In dewetting bilayers, for example, it is often the goal to determine the interfacial energy or the Flory parameter between the two polymers. The contact angle, which depends on the interfacial energies between the two polymers, can in principal be determined from the slope of AFM topographs. ${ }^{8-10}$ However, this is a very indirect method since force or friction modulation contrast is only a reflection of the mechanical response of the sample and is not an unambiguous identification of chemical composition at the interface. Furthermore, if the polymers are in the liquid state then the Newman, rather than the Young's contact angle, must be determined $^{9}$ which requires a precise knowledge of the curvature of the polymer/polymer interface. We present here the combination of near-edge x-ray absorption fine structure (NEXAFS) imaging in transmission ${ }^{11,12}$ and surface sensitive modes $^{13,14}$ as a new approach to characterize polymer thin films and investigated model thin films of blends and bilayers of polystyrene (PS) and brominated polystyrene ( $\mathrm{PBrS})$.
The PS/PBrS system is difficult to study by other techniques and represents a more general case than the polymer systems studied previously with AFM. No mechanical or friction differences can be detected between the two polymers in scanning force microscopy and the bromine content is insufficient to stain one of the phases in transmission electron microscopy (TEM). Our data provide important information about the three-dimensional morphology of the systems investigated, and show that the PBrS gets encapsulated by PS in both blends and bilayers after annealing even in the case where the PBrS started out as the surface layer.

NEXAFS imaging of polymers has advantages over other forms of microscopy primarily because of its low damage, ${ }^{11,15}$ sufficient chemical sensitivity to distinguish essentially most, if not all, polymers, ${ }^{16,17}$ and the possibility for quantitative analysis. ${ }^{18}$ Here we explore explicitly the advantages of combining surface sensitive and bulk sensitive data acquisition modes of $\mathrm{x}$-ray microscopy. We also demonstrate quantitative mapping of composition in thin synthetic polymer films with transmission NEXAFS microscopy, and discuss the implications for the three-dimensional morphology of the systems investigated. We have utilized the Stony Brook scanning transmission X-ray microscope (STXM) ${ }^{19}$ to measure the bulk composition of thin films as thick as 500 $\mathrm{nm}$. In order to assess the surface composition, we have utilized a photoelectron emission microscope (PEEM) at beamline 8.0 at the Advanced Light Source in Berkeley. The electron optical column of the microscope is used to form an image of the emitted electrons. ${ }^{20} \mathrm{We}$ investigated blend and bilayer samples of highly brominated polystyrene (79\% degree of Bromination) and polystyrene (PS). The blends (50/ $50 \%$ by weight, molecular weight $M w=90 \mathrm{k}$ each) were spun cast from toluene solution onto a native oxide covered 


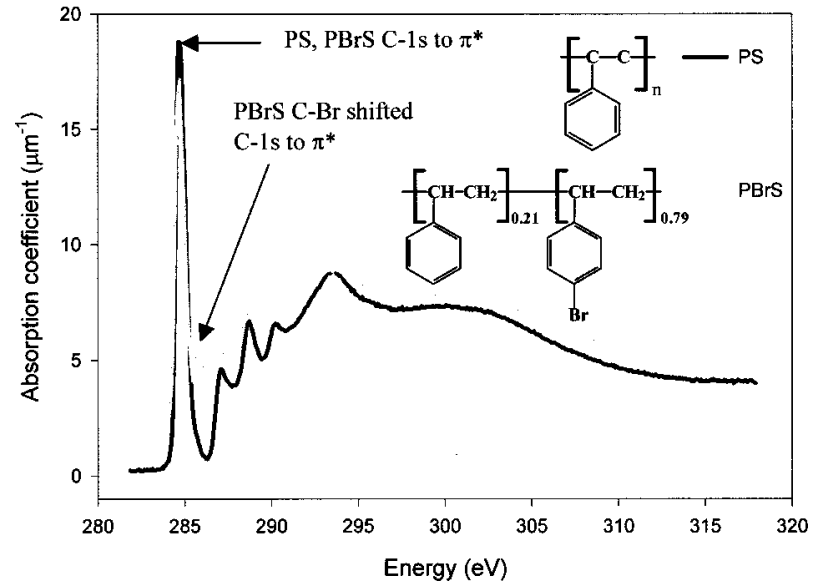

FIG. 1. Reference spectra of PS and PBrS normalized to the pre- and postedge linear absorption coefficients of Henke [B. L. Henke, E. M. Gulikson, and J. C. Davis, At. Data Nucl. Data Tables 54, 181 (1993)], and assuming a density of $1.07 \mathrm{~g} / \mathrm{cm}^{3}$ for PS and $1.53 \mathrm{~g} / \mathrm{cm}^{3}$ for PBrS [J. E. Mark, Physical Properties of Polymers Handbook, Woodbury, New York (1996)].

Si substrate. The film thickness was monitored with multiple wavelength ellipsometry. The bilayers were prepared by spin casting the PS and PBrS separately onto silicon substrates and then floating the PBrS layer on top of the PS layer. The samples were annealed on the substrate under vacuum to initiate the dewetting in the bilayer or to further increase the phase separation in the blends. The samples investigated with PEEM were left on the Si substrate, while the samples investigated with STXM were transferred to a TEM grid. The blends investigated had a thickness of about $45 \mathrm{~nm}$, while the bilayer were $30 \mathrm{~nm}$ of $\mathrm{PBrS}$ on top of $30-40 \mathrm{~nm}$ thick PS.

Homopolymer films were utilized to measure reference spectra as the basis for the quantitative NEXAFS analysis. The linear absorption coefficients of the two polymers are shown in Fig. 1. Regarding bulk compositional analysis, we show as an example the results from a $30 \mathrm{~nm} / 40 \mathrm{~nm} \mathrm{PBrS} / \mathrm{PS}$ bilayer annealed for one week at $180^{\circ} \mathrm{C}$. We acquired STXM images of the same sample area at four characteristic energies: $281.8 \mathrm{eV}$ (pre-edge), $285.2 \mathrm{eV}$ ( $\pi^{*}$ of PS, main $\pi^{*}$ of $\mathrm{PBrS}), 286.5 \mathrm{eV}$ (C-Br shifted $\pi^{*}$ of $\left.\mathrm{PBrS}\right)$, and $310 \mathrm{eV}$ (post-edge). Each image contains information about the PS and $\mathrm{PBrS}$ composition and thickness, with the weight governed by the linear absorption coefficient of the two polymers (Fig. 1). We have utilized a "singular value decomposition" procedure ${ }^{18}$ to calculate PS and PBrS mass thickness maps from the transmission images. This process makes use of the carefully measured absorption coefficients from the reference homopolymers (Fig. 1).

The quantitative PS, PBrS, and total thickness (sum of PS and PBrS) maps are shown in Figs. 2(a), 2(b), and 2(c), respectively. From the figures, we can see the characteristic Voronoi tessellation dewetting patterns that form prior to droplet formation. ${ }^{21}$ The respective line profile as indicated in the images is shown directly below each figure. From Fig. 2(a), we can see that a PS ridge surrounds the interconnecting strands of the $\mathrm{PBrS}$ layer. The $\mathrm{PBrS}$ core within the strands is seen in Fig. 2(b) to rise much more sharply. Consequently, it is clear that the contact angle between the PS and $\mathrm{PBrS}$ features cannot be measured from the topographic

Downloaded 19 Feb 2008 to 152.1.211.43. Redistribution subject to

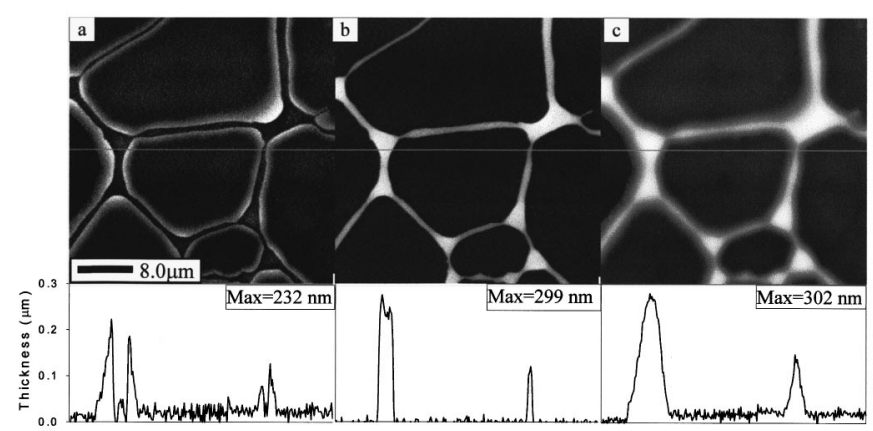

FIG. 2. Thickness maps of PBrS/PS bilayer after annealing for 1 week: (a) PS map, (b) PBrS map, and (c) total thickness.

features alone. Even though the dewetting process has not reached equilibrium yet, we estimate the contact angle from the cross sectional scan to be $\sim 10^{\circ}$ when measured from the PS and total density maps in Figs. 2(a) and 2(c). This value is in agreement with that obtained from atomic force microscopy of the sample before removal from the substrate. The contact angle of the PBrS core on the other hand is $\sim 20^{\circ}$ which is closer to the calculated value of $28^{\circ}$ based on measurements of the interfacial energy of the two polymers. ${ }^{22}$

In order to address the issue of encapsulation, we determined the surface composition of a similar sample, i.e., a 30 $\mathrm{nm} / 34 \mathrm{~nm}$ PBrS/PS bilayer annealed for 11 days. PEEM images acquired at $300 \mathrm{eV}$ are presented in Fig. 3 and show Voronoi tessellation patterns ${ }^{21}$ similar to the ones observed in Fig. 2 with transmission NEXAFS. The sides of the spines and the spines themselves are dark due to topographic contrast that arises from the reduced collection efficiency in the PEEM from locations that are not perpendicular to the optical axis. We present spectra in Fig. 4 from various locations on the samples, including several locations along the spines, as indicated by the boxes in Fig. 3. While these spectra have not been normalized to the carbon edge continuum, they clearly show that there is no spectral intensity at the $\mathrm{C}-\mathrm{Br}$ shifted $\mathrm{C} 1 s$ to $\pi^{*}$ energy of $286.3 \mathrm{eV}$ that is characteristic of $\mathrm{PBrS}$. Hence, the surface is pure PS everywhere on the

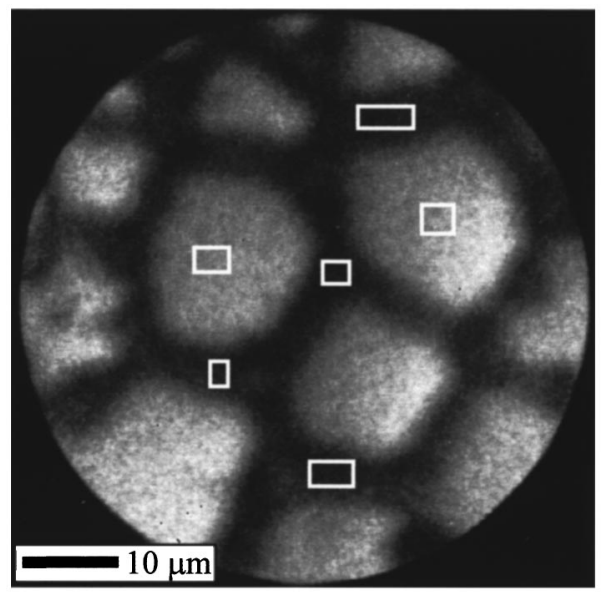

FIG. 3. PEEM image of a $30 \mathrm{~nm} / 34 \mathrm{~nm}$ PBrS/PS bilayer annealed for 11 days (acquired at $300 \mathrm{eV}$ ). A Voronoi tesselation pattern similar to Fig. 2 is clearly visible. The differences in appearance relative to Fig. 2 are due to the different contrast mechanism in a PEEM and a STXM. The boxes indicate the areas of which the spectra in Fig. 4 where acquired. The arrow bar equals $20 \mu \mathrm{m}$.

AlP license or copyright; see http://apl.aip.org/apl/copyright.jsp 


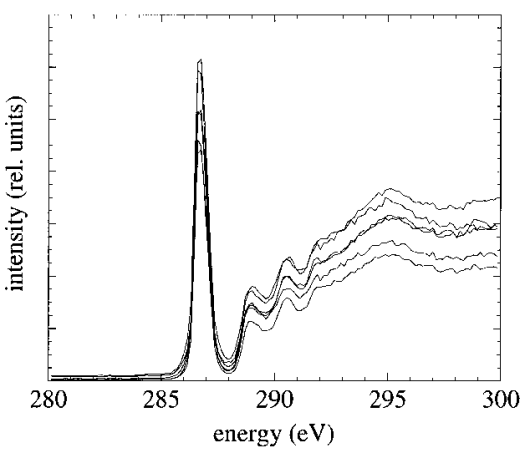

FIG. 4. Point spectra acquired with the PEEM from various locations on the sample as indicated by the boxes in Fig. 3 .

sample within the characteristic probing depth of NEXAFS PEEM of about $10-20 \mathrm{~nm}$. We have also found that the $\mathrm{PBrS}$ is encapsulated in blends that have been annealed. The encapsulation of $\mathrm{PBrS}$ is consistent with the thermodynamically favored state where PS, the component with the lower surface energy segregates to the film surface. ${ }^{4}$

In general, the dewetting process in thin polymer bilayers can consist of diffusive and flow processes both parallel and perpendicular to the polymer/polymer interface. We can obtain an estimate of the relative magnitude of these processes, i.e., diffusion/flow of the PBrS and PS along the $\mathrm{PBrS} / \mathrm{PS}$ interface and PS perpendicular to the interface from Fig. 5, where we show area averaged PEEM spectra of bilayer samples annealed for 1 day, 2 days, and 11 days. These spectra clearly show that encapsulation does not occur immediately at the onset of dewetting. After two days of annealing, the PBrS layer has already moved parallel to the PS interface to form holes, several microns in diameter, exposing the underlying PS at numerous places. The lateral distance traveled by the PBrS along the polymer/polymer interface is much larger than the PBrS film thickness $(\sim 40 \mathrm{~nm})$, yet the PS encapsulation, which requires perpendicular diffusion over a distance that is approximately the thickness of the $\mathrm{PBrS}$ overlayer, has not yet occurred. This is illustrated in Fig. 5, where we can see that the area-averaged spectrum after 2 days is still dominated by the PBrS overlayer. Encapsulation is essentially complete after annealing for 11 days (Fig. 4). This indicates that the diffusion/flow parallel to the interfaces is much faster for both PS and PBrS than the diffusion of PS perpendicular to the PS/PBrS interface. A possible explanation for this phenomenon may be that since the $\mathrm{PS}$ and $\mathrm{PBrS}$ are highly immiscible, the diffusion of PS through the PBrS layer is severely hindered. The optimum

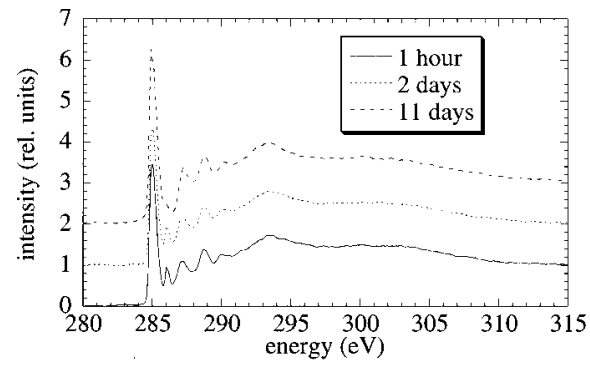

FIG. 5. Evolution of PBrS peak in PEEM spectra from a $30 \mathrm{~nm} / 35 \mathrm{~nm}$

PBrS/PS bilayer as a function of annealing time.
Downloaded 19 Feb 2008 to 152.1.211.43. Redistribution subject to AIP license or copyright; path for encapsulation is then by PS diffusion/flow along the outer surfaces of the dewetting features. Hence the encapsulation process seems to be delayed until the dewetting of the PBrS layer from the PS interface has progressed sufficiently that a significant amount of exposed PS surface area has formed.

In summary, we have used the unique combination of two complementary techniques, PEEM and STXM NEXAFS microscopy, to determine the three-dimensional composition of polymer thin films. This allowed us to study both the surface and bulk morphology of PS/PbrS blends and the dewetting dynamics of PS/PbRS bilayers. Our results show that PBrS forms sharply delineated spines that become encapsulated in PS as the PBrS dewets the PS support film. The $\mathrm{PS} / \mathrm{PBrS}$ contact angle is much larger than the contact angle measured with AFM of the external topographical features.

The STXM data were acquired with the Stony Brook STXM at the National Synchrotron Light Source. The PEEM data were acquired at the Advanced Light Source. H. Ade, D. A. Winesett, and A. P. Smith are supported by NSF Young Investigator Award No. DMR-9458060. M. H. Rafailovich and J. Sokolov are supported by NSF DMR-9732230 (MRSEC Program) and DOE-SG02-93-ER45481.

${ }^{1}$ J. Genzer and E. J. Kramer, Phys. Rev. Lett. 78, 4946 (1997)

${ }^{2}$ S. Affrossman, G. Henn, S. A. O'Neill, R. A. Pethrick, and M. Stamm, Macromolecules 29, 5010 (1996).

${ }^{3}$ E. Kumacheva, L. Li, M. A. Winnik, D. M. Shinozaki, and P. C. Cheng, Langmuir 13, 2483 (1997).

${ }^{4}$ G. Krausch, E. J. Kramer, M. H. Rafailovich, and J. Sokolov, Appl. Phys. Lett. 64, 2655 (1994).

${ }^{5}$ W. Straub, F. Bruder, R. Brenn, G. Krausch, H. Bielefeldt, A. Kirsch, O. Marti, J. Mlynek, and J. F. Marko, Europhys. Lett. 29, 353 (1995).

${ }^{6}$ K. Tanaka, A. Takahara, and T. Kajiyama, Macromolecules 29, 3232 (1996).

${ }^{7}$ S. Wahlheim, M. Boltau, J. Mlynek, G. Krausch, and U. Steiner, Macromolecules 30, 4995 (1997).

${ }^{8}$ P. Lamboony, K. C. Phelan, O. Haugg, and G. Krausch, Phys. Rev. Lett. 76, 1110 (1996).

${ }^{9}$ S. Qu, C. J. Clarke, Y. Liu, M. H. Rafailovich, J. Sokolov, K. C. Phelan, and G. Krausch, Macromolecules 30, 3640 (1997).

${ }^{10}$ K. R. Shull and T. E. Karis, Langmuir 10, 334 (1994).

${ }^{11}$ H. Ade, X. Zhang, S. Cameron, C. Costello, J. Kirz, and S. Williams, Science 258, 972 (1992).

${ }^{12}$ X. Zhang, H. Ade, C. Jacobsen, J. Kirz, S. Lindaas, S. Williams, and S. Wirick, Nucl. Instrum. Methods Phys. Res. A 347, 431 (1994).

${ }^{13}$ A. Cossy-Favre, J. Diaz, S. Anders, H. A. Padmore, Y. Liu, M. Samant, J. Stohr, H. Brown, and T. P. Russel, Acta Phys. Pol. A 91 (1997).

${ }^{14}$ A. Cossy-Favre, J. Diaz, Y. Liu, H. Brown, M. G. Samant, J. Stöhr, A. J. Hanna, S. Anders, and T. P. Russell, Macromolecules 31, 4957 (1998).

${ }^{15}$ E. G. Rightor, A. P. Hitchcock, H. Ade, R. D. Leapman, S. G. Urquhart, A. P. Smith, G. Mitchell, D. Fisher, H. J. Shin, and T. Warwick, J. Phys. Chem. B 101, 1950 (1997).

${ }^{16} \mathrm{~J}$. Stöhr, NEXAFS Spectroscopy (Springer, Berlin, 1992).

${ }^{17}$ H. Ade, Trends Polym. Sci. 5, 58 (1997).

${ }^{18}$ X. Zhang, R. Balhorn, J. Mazrimas, and J. Kirz, J. Struct. Biol. 116, 335 (1996).

${ }^{19}$ C. Jacobsen, S. Williams, E. Anderson, M. T. Brown, C. J. Buckley, D. Kern, J. Kirz, M. Rivers, and X. Zhang, Opt. Commun. 86, 351 (1991).

${ }^{20}$ B. Tonner, D. Dunham, T. Droubay, and M. Pauli, J. Electron Spectrosc. Relat. Phenom. 84, 211 (1997).

${ }^{21}$ T. G. Stange, R. Mathew, D. F. Evans, and W. A. Hendrickson, Langmuir 8, 920 (1992).

${ }^{22}$ W. Zhao, X. Zhao, M. H. Rafailovich, T. M. J. Sokolov, R. S. Stein, R. C. Composto, E. J. Kramer, R. A. L. Jones, M. Sansone, and M. Nelson, Physica B 173, 43 (1991). 